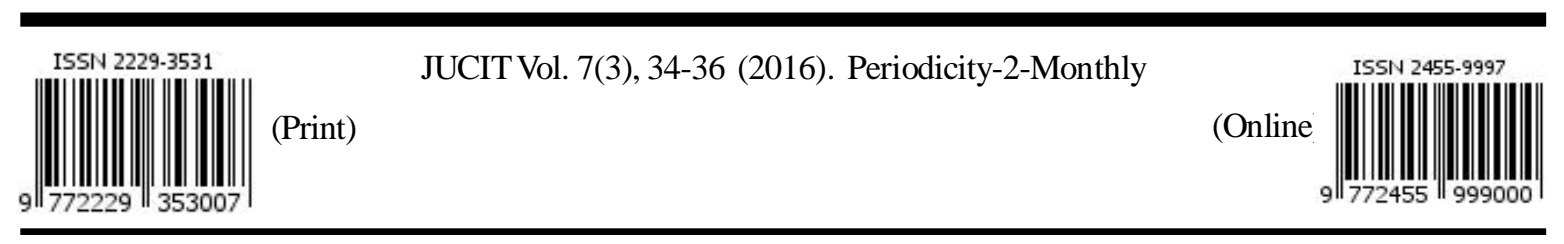

JOURNAL OF ULTRA COMPUTER \& INFORMATION TECHNOLOGY
An International Open Free Access Peer Reviewed Research Journal of Computer
Science Engineering \& Information Technology
website:- www.compitjournal.org

\title{
A Review of Image Compression for Medical Images Using Differential Pulse Code Modulation (DPCM)
}

\author{
MITHIL KULHARA ${ }^{1}$ and PANKAJ KUMAR SINGH ${ }^{2}$ \\ ${ }^{1}$ M. Tech Scholar, Department of Computer Science \& Engineering, SISTECC-R Bhopal (M.P), India \\ ${ }^{2}$ Assistant Professor Department of Computer Science \& Engineering, SISTECC-R, Bhopal (M.P), India \\ ${ }^{1}$ Email of Corresponding Author:- mithi12401@gmail.com, \\ http://dx.doi.org/10.22147/jucit/070302
}

Acceptance Date 7th December, 2016,

Online Publication Date 28th December 2016

\begin{abstract}
Image processing modifies pictures to improve, extract information and change their structure (composition, image editing and image compression etc.). Image can be processed by optical, photographic and electronic means, but image processing using digital computers are the most common methods because digital methods are fast, flexible and precise. Image Compression- reducing the redundancy in the image data to optimize transmission / storage. This paper outlines the different types of image compression and lastly we compare the Differential Pulse Code Modulation (DPCM).
\end{abstract}

Keywords: EDPCM, Compression Ratio (CR), Encoding Techniques

\section{Introduction}

The objective of image compression is to reduce the redundancy of an image. Lossless compression is to reduce the redundancy of an image. Lossless compression method applies when data are critical and loss information is not acceptable. Medical image compressions are based on lossless compression method. Medical imaging is been used for diagnosis of diseases and surgical planning, and they need long-term storage for profiling patient's data as well as efficient transmission for long diagnosis ${ }^{1}$. It is essential to make the medical image compression lossless to avoid of critical medical information. In the field of online diagnosis or real time applications such as telemedicine, demands for hardware to handle lossless compression that can accelerate the computation process. There have been many studies on medical lossless compression algorithm. DPCM has an advantage over other lossless compression schemes due to simple structure to implement ${ }^{2}$.

\section{Problem Formulation :}

The Objective of this proposed work is to implement a robust technique that works for the images compression using Differential Pulse Code Modulation (DPCM) which can compress the data as much as possible. In this multimedia edge efficient compression is really a tuff work. The early research in image compression introduced many techniques such as JPEG, JPEG-2000, and JPEG-LS.

3Image Processing Techniques:

This is an open access article under the CC BY-NC-ND license (https://creativecommons.org/licenses/by-nc-sa/4.0) 
The analysis and manipulation of a digitized image, especially in order to improve its quality, storage \& transmissions in networks or cloud. Our aim is how to improve compression techniques for implementing the above requirements. Compression is useful because it helps reduce the consumption of expensive resources, such as hard disk space or transmission bandwidth. On the downside, compressed data must be decompressed to be used, and this extra processing may be determined to some applications. Data compression can be defined as reducing of the amount of storage space required to store a given amount of data ${ }^{3}$.

Data compression comes with a lot of advantages. It saves storage space, bandwidth, cost and time required for transmitting data from one place to another. Compression can be lossy or loss-less. With a loss-less compression and decompression the original and decompressed files are identical bit per bit. On the other hand, compression efficiency can be improved by throwing away most of redundant data, without however losing much quality.

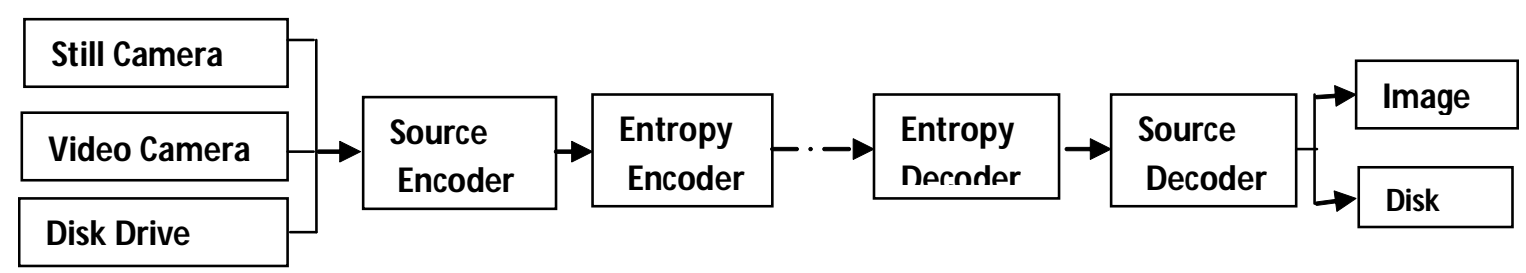

Figure1. Source Encoding and Decoding of image or video data for Storage or Transmission ${ }^{5}$

There are many loss-less compression technique such as Arithmetic coding, Run Length Encoding, Huffman coding and some famous Dictionary based algorithm like Lempel-Ziv- Welch (LZW) coding, through Huffman coding forms the basis of many compression algorithms. JPEG, MPEG, which are lossy compression methods use Huffman coding. Even the new proposal algorithm like JPEG-2000 Burrows-Wheeler transformation (Bwt) and BTTC use Huffman coding in the final stage ${ }^{4}$.

\subsection{Types of Image Compression:}

There are 2 types of image compression: lossless compression (reversible) and lossy compression (irreversible). Run-length encoded (RLE) and the JPEG lossless compression algorithms are example of lossless compression.

In lossy compression, data are discarded during compression and cannot be recovered. Lossy compression achieves much greater compression than lossless techniques ${ }^{5}$. Wavelet and higher-level JPEG are examples of lossy compression. JPEG 2000 is a progressive lossless-to-lossy compression.

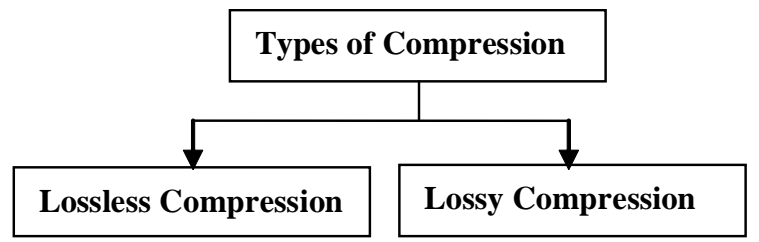

Figure 2. Types of Image Compression

\subsubsection{Lossless Image Compression:}

When hearing that image data are reduced, one could expect that automatically also the image quality will be reduced. A loss of information is, however, totally avoided in lossless compression, where image data are reduced while image information is totally preserved ${ }^{6}$. It uses the predictive encoding which uses the gray level of each pixel to predict the gray value of its right neighbour. Only small deviation from this prediction is stored. This change the statistics of the image signal drastically. Statistical encoding is another important approach to lossless data reduction. Statistical encoding can be especially successful if the gray level statistics of the images has already been changed by predictive coding. The overall result is redundancy reduction that is reduction of the reiteration of the same bit patterns in the data. Losslesscompression is therefore also called reversible compression.

\subsubsection{Lossy Image Compression :}

Lossy data compression has of course a strong negative connotation and sometimes it is doubted quit emotionally that it is at all applicable in medical imaging.

In transform encoding one performs for each image run a mathematical transformation that is similar to the Fourier transform thus separating image information on gradual spatial variation of brightness (regions of essentially constant brightness) from information with faster variation of brightness at edges of the image.

In image data reduction, this second step is called quantization. Since this quantization step cannot be reversed when decompressing the data, the overall compression is 
'lossy’ or ‘irreversible’.

\section{Proposed Methodology:}

The proposed technique uses Differential Pulse Code Modulation (DPCM) for image compression in efficient manner. In this method we have two system, Compression system and Decompression system. Since we know that we have many techniques to implement transformation. Here we will use Differential Pulse Code Modulation.

For Encoding we have many techniques but we will try to use Huffman Encoding because it is efficient and easy to implement. During Reconstruction of image we have to apply both Transformation and Encoding in Reverse manners.

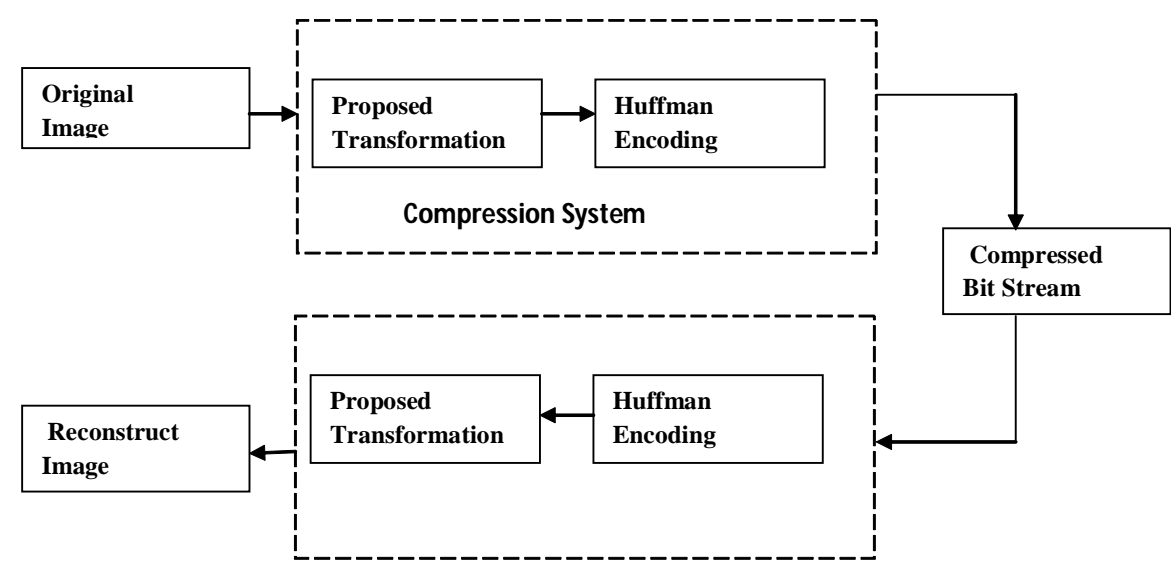

Figure 3. Propose Method Block Diagram

\section{Conclusion}

In this paper, we analyses the previous work done in the image compression using various Transformation and encoding techniques and we find that Differential Pulse Code Modulation (DPCM) and Huffman encoding has better performance and easily to implement.

\section{References}

1. S. Burak, C. Tomasi, B. Girod and C. Beaulien, "Medical Image Compression based Region of Interest with Application to Colon CT images," Processing of 23rd International Conference of the IEEE Engineering in Medicine and Biology Society, Istambul, Turkey (2001).

2. L. Zhao,Y. Titan, Y. Sha and J. Li, "Medical Image Lossless Compression base on Combining an Integer Wavelet Transform with DPCM," Frontiers of Electrical and Electronic Engineering, China on (2009).

3. S. Jayaraman, S. Esakkirajan, T. Veerakumar, "Digital Image Processing, TMH Publication," pp. 444-542, Year of Publication (2009).

4. Rafael C. Gonzalez, Richard E. Woods, Steven L.
Eddins, "Digital Image Processing Using Matlab, Low Price Edition," pp.1-10, 374-438, Year of Publication (2010).

5. Adina Arthur, V. Saravanam, "Efficient Medical Image Compression Technique for Telemedicine Considering online and Offline Application," Computing, Communication and Applications (ICCCA), 2012 International Conference on Feb (2012).

6. M.J. Weinberger, G. Seroussi and G. Saprio, "LOCO-1 Lossless Image compression Algorithm: Principles and Standardization into JPEG-LS,” Image Processing, and IEEE Transactions on Aug (2000).

7. Ranbeer Tyagi, D. K. Sharma "Digital Image Compression Comparisons using DPCM and DPCM with LMS Algorithm," International Journal of Computer Applications \& Information Technology Vol. I, Issue II, September (2012).

8. B. P. Lathi and Zhi ding "Modern Digital and Analog Communication Systems" International Fourth Edition. New York Oxford University Press-2010, pp.292.

9. J. E. Modestino and D. G. Daut, "Source-channel coding of images," IEEE Communication Society, vol. COM27, pp. 1644-1659, Nov. (1979). 